\title{
Staging Blockchain Technology In The Customer's Journey Through Service Design
}

\author{
A Bike-sharing Case Study To Improve Quality And Trust Perception \\ Evelyn Durugy ${ }^{\dagger}$ \\ Institut of Management \\ University of Neuchâtel \\ Neuchâtel Neuchâtel Switzerland \\ evelyn.durugy@googlemail.com \\ Oussama Darouichi \\ Institut of Management \\ University of Neuchâtel \\ Neuchâtel Neuchâtel Switzerland \\ oussama.darouichi@unine.ch \\ Emmanuel Fragniere \\ HES-SO Valais-Wallis \\ and University of Bath \\ Sierre Valais Switzerland \\ emmanuel.fragniere@hevs.ch
}

\begin{abstract}
Thanks to the growing trend of the sharing economy and the increased need for innovative mobility solutions, the bike sharing sector has gained considerable importance. However, the privacy of the participants is at risk as their user and travel profiles are not only collected and used by bike sharing service providers. In addition, for the bike share provider, problems have arisen due to increased vandalism and theft of the vehicles on offer. Therefore, this experimental study shows why Blockchain could solve this problem by eliminating dependency on third parties and allowing an economical and user-friendly mode of transport between participants directly. As part of this research, we have "staged" the blockchain through service design in the context of bike sharing. Blockchain will only demonstrate its value when integrated into real service experiences. Our main conclusion is that "blockchain staging" is more important than the technological dimension of smart contracts.
\end{abstract}

\section{CCS CONCEPTS}

- Human-centered computing Visualization Empirical studies in visualization $•$ Human-centered computing Interaction design Empirical studies in interaction design

\section{KEYWORDS}

Blockchain technology, Human-centric approaches, Service design, Sharing economy

\section{ACM Reference format:}

Evelyn Durugy, Oussama Darouichi and Emmanuel Fragniere. 2020. Staging Blockchain Technology In The Customer's Journey Through Service Design: A Bike-sharing Case Study To Improve Quality And Trust Perception. In Proceedings of the International Conference on Industrial Control Network and System Engineering Research (ICNSER'20). Kuala Lumpur, Malaysia, 7 pages.

https://doi.org/10.1145/3411016.3411161

${ }^{\dagger}$ Corresponding Author

Permission to make digital or hard copies of part or all of this work for personal or classroom use is granted without fee provided that copies are not made or distributed for profit or commercial advantage and that copies bear this notice and the full citation on the first page. Copyrights for third-party components of this work must be honored. For all other uses, contact the owner/author(s).

ICNSER'20, June, 2020, Kuala Lumpur, Malaysia

(C) 2020 Copyright held by the owner/author(s). $978-1-4503-7549-8 / 20 / 05 \ldots \$ 15.00$ https://doi.org/10.1145/3411016.3411161

\section{Introduction}

The Blockchain technology is considered by many to be a revolution in the business world. Theoretically, the blockchain could lead to a situation where no intermediary is needed for any economic transaction. Thanks to cryptographic algorithms and an almost infinite number of replications on computers around the world, a blockchain contract could never be altered, hidden or cancelled. As a result, trust in those acting as intermediaries would no longer be necessary [8]. A new trust would therefore be created through blockchains. But these blockchain technologies have to prove their usefulness not at an abstract or conceptual level, but at the level of the business experience between a customer and a supplier [5]. In particular, our traditional business models, which are ultimately very linear, i.e. B2B and B2C, could possibly be complemented by a variety of new provider-user relationships, with new models such as $\mathrm{C} 2 \mathrm{C}$ and even $\mathrm{C} 2 \mathrm{~B}$ being addressed in the co-production and co-customer logic. Within the framework of this paper, we develop through service design [20] a business model of shared economy integrating blockchain applied to the case of soft mobility (i.e. bike sharing).

The main question we want to address is why the blockchain can bring a real advantage to the cycling experience in a shared economy framework as well as how to integrate the blockchain technology pragmatically and reliably in purely digitalized management systems. This question is contextualized, taking into account elements of sharing economy, bike-sharing service and blockchain technology as well as references to the scientific literature. Our service design methodology starts from a persona, hypothetical scenarios of practical problems that this persona may encounter in his typical customer journey, for example, on his/her way to work. Then, this customer journey involving "fail points" is integrated into "service blueprinting" to relate the customer journey to the different touchpoints and also to the touchpoints related to the use of the bike. Finally, a real simulation is conducted, and pragmatic solutions are proposed to establish a practical link with the blockchain technology. We conclude this paper with a discussion around a model we have developed based on our simulations. 


\section{Literature review}

\subsection{Sharing Economy and Ownership}

The consumer behavior of today is significantly undergoing a process of change. The typical consumer who just a few years ago overconsumed is now starting to change his consumption behavior. Instead of acquiring ownership of things, the new consumer aims to achieve a "just-in-time" availability of commodities. For the temporary -possession of things is usually just as effective as their ownership - without having to bear the associated burdens [3].

Physical things take on a new significance, especially after the last financial crisis in 2009, were the citizen started to leave the age of hyper-consumption towards mindful consumption. As a result, a business model has emerged that allows consumers to get what they want and need at lower personnel and environmental costs. A business model which would even have the power to replace prevailing economies and mainstream consumerism [9]. This new economic force is known as the sharing economy.

Sharing economy, which was promoted by innovative information and communication technology, allowed to open up a new way of coordination of demand and supply of goods and services. Thus, as we have distinguished between the forms of participants, there is also a difference in the notion of ownership in relation to the sharing economy. The sharing activity takes place either between different users without transfer of property in the sense of intensification of use (lending, renting, shared use) or with the transfer of ownership in the spirit of extension of useful life (selling, giving away, exchanging). The different transactions of the sharing economy can be divided into the temporary (shortterm- or complete ownership transfer) and the service which is provided (lending, renting, shared use, selling, giving away, or exchanging) [2].

\subsection{Bike Sharing and Issues}

Bikes sharing systems [14] works as follows. A supplier distributes a fleet of bikes across the city in the form of either stationed or free-floating bikes. People interested in using the service will have to register with the selected provider with their personal data, including their payment details. A credit card or bank card usually makes the payment, and the connection data is stored on the network when the user registers. Bike-sharing providers are charging fees for the usage and eventually earn extra for advertisement they show on their bikes.

Although bike-sharing providers decline that they are selling and using client's data, data protection agencies are concerned. The assumption behind this is that some businesses are in reality targeting the user profiles of their cycling customers - for example, to use them for personalized advertising [13].

Bike sharing has become common in many places, an essential component of municipal strategies for sustainable urban mobility concepts. If the rental bikes are correctly positioned in the city and available in sufficient numbers, they supplement public transport and replace part of the car traffic. For a long time, the bikesharing market (this actually the case in Germany) was dominated by station-based providers. At present, however, systems without fixed parking facilities, the so-called free-floating model, are developing to offer users as much flexibility as possible. This means flexibility for the user, means on the other side chaos on the streets. Since the introduction of the free-floating models, cities have been complaining about the arbitrary parking of bicycles. Because the bikes have no fixed stations, providers can simply distribute them in public spaces without special permission from the city. As bicycles are neither ownerless nor municipal property, cities and municipalities are not allowed to store them themselves. It is the user who causes this distribution problem and inadequate parking when focusing on the floating model. The mentality "what I don't own doesn't matter to me," takes over more and more.

Bike-sharing providers also have to struggle immensely with vandalism and theft. In some cities, it is so blatant that bikesharing providers are consistently leaving certain areas or even countries in order to avoid economic losses like it was the case with Globee, a bike-sharing company that has announced its exit from the French market. The reason for it was the mass destruction of its own bicycle fleet. Around 3,400 rental bicycles were damaged, and 1,000 stolen [16]. This, in turn, is counterproductive for the desired green mobility. There are already proposals for solutions such as surveillance cameras attached to bicycles, but this would contradict the extreme of data protection, which is strictly adhered to, for example, in Germany. We see that the sharing economy and the bike sharing model mentioned above also pose problems that are based on external factors, whether it is the company itself, which is trying to use user data to run a profitable business with the mega-trend of the sharing economy in combination with mobility, or whether it is the user himself who is working counterproductively for the general welfare of society by leaving bikes in inappropriate places where they annoy others [15].

Some of them even destroy or steal bikes out of personal intention. As there is no real punishment for the user, bike-sharing providers are helpless. Therefore, this case study will focus on the problems which arose over the years targeting the bike-sharing sector. The idea is to find a solution to how user behaviour could change with regard to distribution, vandalism and theft, and how it is possible to eliminate the manipulation of user data and dependence on bike sharing service providers.

\subsection{Blockchain and Sharing Economy}

After the turn of the century, the Internet seemed less and less suitable for transferring sensitive valuables such as money, data or, property rights. The development of blockchain technology could change that as the main feature of this technology is the settlement of secure transactions between two partners without them having to trust each other.

The blockchain technology makes central instances superfluous by transferring their functions to all participants of a system. It functions like a decentralized booking system that is managed collectively according to transparent rules via the computers of all participants. Each participant in the blockchain network can 
transmit new changes (transactions) to the database. However, they are only accepted if they are permissible or if they do not compromise the database. This means that no participant can change the entries of other participants. To control this, each participant in the blockchain system can view a copy of all transactions in the entire database at any time to track any transaction. So, there is no potential corrupt central instance that can be hacked or bribed. Precisely because the blockchain does not belong to anyone or belongs to everyone, anyone can trust it [1]. Ownerless platforms could make new business models and a true sharing economy possible. Blockchain paves the way for usage-based business models. So, bike-sharing providers and other participants like Uber and Airbnb could take away the high placement fees and still use the transmitted personal data of the users for them or pass them on to third parties. With a blockchain, the same platform can be set up without intermediate commercial instances. Only that all processes would be faster, cheaper, and more secure against data protection violations. In this sense, through blockchain, it is possible to enable the exchange of bikes in-between the users directly, and therefore create a real peer-topeer network without relying on a business. Blockchain brings new challenges with it. One of them is the understanding of technology. If the blockchain is to develop its full potential, people need to understand how it works. And therefore, it is essential to develop real use cases. And thus, build up a concept of a real peer-to-peer bike-sharing system by using smart contracts and eliminate any third-party providers. Service design approaches are therefore essential since the entire service experience must be based directly on the users. The question that arises is whether a model of bike sharing without any intermediary would work and be successful, and how it can be put into practice.

\section{Service Design Methodology: Blueprinting}

In this paper, we rely service design techniques such as the persona, the Customer Journey Map (CJM) and the service blueprint [10] that are mapping tools used in service design to visualize organizational processes in order to optimize how a business delivers a user experience. Practically, a service experience corresponds to a social process whose "production" involves both a provider and a client [6]. Based on a given customer journey integrated in a service blueprint, different customer touchpoints are shown up which represent intersections between the service provider and its users. A service acquires value once the user perceives its benefits [6]. An element of perceived value in service design is called a salient attribute. These attributes are perceived simultaneously at the moment the service is produced. "An attribute is salient for a good when it stands out among the good's attributes, relative to that attribute's average level in the choice set." [4].

In order to integrate targeted enhancements directly in the user experience, we first outline a rough customer journey followed up by a more precise blueprint to identify given vertices of the improved service. This technique in service design is called zooming in/zooming out. Once a touchpoint of the blueprint has been improved (zooming in), we step back (zooming out) to keep an overview of the entire service experience. Here, the term "service" is identified as a process of co-creation between a client/user and a producer. A given service experience is considered heterogeneous because of the unique interactions between the customer and the producer [19]. Applying this in our methodology, we need to identify the essential elements of the service experience and then sketch out the user's journey that they must go through in order to receive the service. The aim hereby is to find out critical touchpoints (also called "fail points") of the user, which will lead us to identify improvement possibilities.

After a series of immersion episodes (based on participative and non-participative observation techniques [16]) in rental and sharing bike services in Switzerland and Germany, we came up with a generic customer journey map (see an example in Figure 4) of what a user should experience without the blockchain technology. We can this way illustrate in a simplified and selfexplanatory manner the individual steps that the user goes through to lend or use a bike via a typical bike-sharing service. This step helped us in displaying the nature and sequence of the different steps in delivering service to customers [12].

Then the service blueprint specifies the received service in more detail and allows the customer's point of view to be combined with that of the provider. Visible but also invisible interactions with the user are registered to point out where possible failure points could arise, and which would result in failure to access or enjoy the complete service experience.

In our blueprints, we have integrated the different stages, which are connected to actions made by users' interactions. These stages show up chronological steps of the process according to their proximity to the user. Three lines are typically set up in the service blueprint [17] (see Figure 1 for an example of blueprint we have developed as part of this research):

-Line of interaction

-Line of visibility

-Line of internal interaction.

Above the line of interaction are the activities in which the customer himself is actively involved, like registering on the webpage, filtering, and riding a bike as well as returning it back. Below this line is the activities performed by the provider and which are in the field of vision of the user. The physical evidence level describes the tools the user is interacting with, like search engines, email forms, or with the bike itself.

Activities below the line of visibility are invisible to the customer ("backstage"). Backstage processes forms are, for example, cookies, automated systems that trigger email send-outs, or processing of customer data for billing purposes. This, in our case, "virtual backstage" is additionally depending on external services like Google, Facebook, insurer, and financial provider like Paypal. 


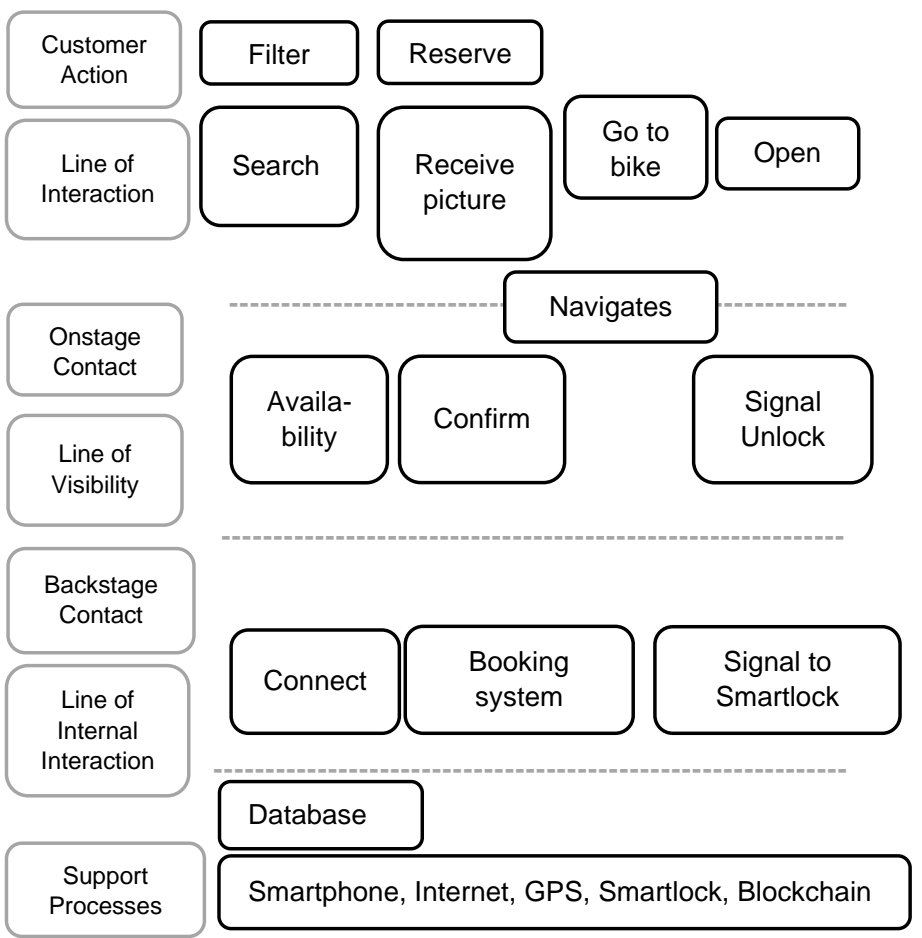

Figure 1: Example of a service blueprint we have used within the context of this research (here a "zooming in")

\section{Feasibility Case study}

\subsection{From Concept to Operationalization}

To operationalize our concept of bike-sharing, including the blockchain technology, we start from a persona and hypothetical scenarios of practical problems that this persona may encounter in his typical customer journey, for example, on his/her way to work. Then, his/her customer journey involving a problem is integrated into a service blueprint to link the customer journey to the different touchpoints and also to the touchpoints related to the use of the bike. Finally, real simulations (involving role-playing) were conducted, and pragmatic solutions proposed to establish a pragmatic link with the blockchain technology.

\subsection{Staging through Simulations}

In our simulations, we have focused on the actual service of the peer-to-peer bike-sharing. Based on our immersion episodes, we have then identified three major critical touchpoints and have created a hypothetical zoomed-in Blueprint for each. We saw challenges with: locating a bike at crowded places, reserved but unsuitable bikes, and access on private property.

The service design visualization tools that we have developed during the whole research period that took place in 2019 (available in an e-companion linked to this paper) is listed below:

1. Initial customer journey based on fieldwork (with no blockchain)

2. Fictitious persona describing a generic user

3. Customer Journey of the new concept (integrating b-blockchain)

4. Blueprint of new concept (zooming out)

5. Blueprint-Bike located at crowded place:

6. Blueprint-Unsuitable bike: zooming in and improvement Blueprint of bike located at a private property: zooming in and improvement

7. Adjusted and improved blueprint of the new concept: zooming out

8. Customer Journey map of the new model

A fictitious persona, called Luis (see Figure 2), was chosen to undergo real-time simulations with the identified theoretical fail points.

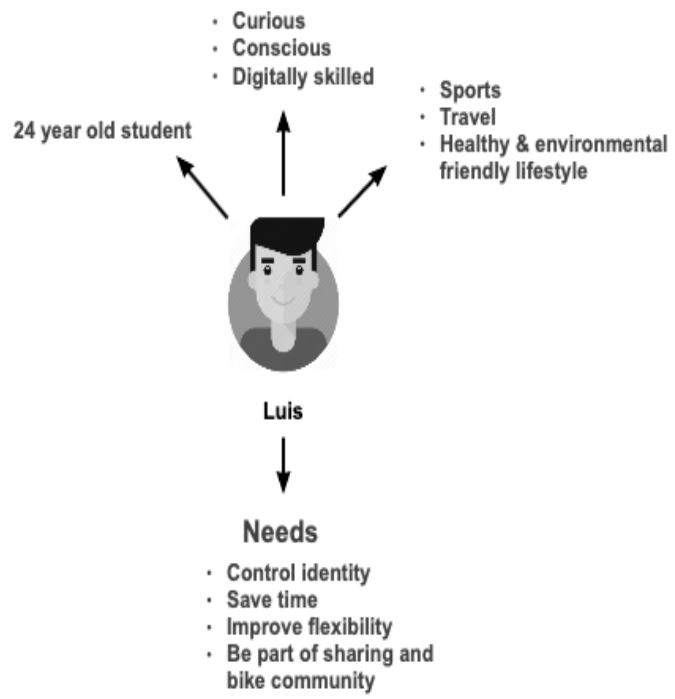

Figure 2: The persona, Luis, employed in the bike-sharing simulation

After each simulation, each participant playing the role of Luis regarding a given blueprint was interviewed based on the four following open questions: 
Staging Blockchain Technology In The Customer's Journey Through Service Design

- $\quad$ Could you describe your experience to me in detail?

- Which aspects did you particularly like?

- Which aspects did you like less or not at all?

- If you had a magic stick, what would you change?

With the results we gained from the fieldwork, we drew up a practical zoomed-in blueprint to evaluate how we could improve the specific problematic. We have then come up with possible solutions that were then tested again with the persona in the same test environment. The test persona was guided through the improved version and the figurant was interviewed again with the same questions.

Here is an excerpt of the narratives that were developed based on audios, photos (see for example Figure 3) and videos of our immersions and simulations related to bike sharing:

"The more bikes are available, the more flexible our user is, and thus, the more satisfied he is. To get to the bike, Luis would be very happy about an integrated navigation system which would bring him to the closest bike. As we have seen in the field test, it is essential for him to have the possibility to filter the size and type of bike to prevent an unpleasant and dangerous ride. In addition, he would be happy about an acoustic signal which would be activated when his chosen bike is located in a crowded place. Our bike-sharing service should also be available in suburbs. As Luis is not living directly in the city, he would be happy to have the opportunity to use on all his last miles a bike and not just in the city where there is already a big offer, whether it is public transport or other bike-sharing providers...

From the synthesis of answers and observations from the three improved fail points, we could then sketch up the overall customer journey (see Figure 4) of the new bike-sharing concept including the blockchain technology and the related zoomed-out final blueprint, which showed us an overall improved service for our concept. If we had neglected this real-time simulation approach and the zooming in/out logic regarding specific situations, we would not have been able to "make sense" of the blockchain technology directly within the user experience.

Let us now look in detail at just one of the three points of failure that we have identified. It corresponds to the problematic in allocating the reserved bike in crowded places, for example, at a train station. This was initially identified in our first immersion episodes and simulations. Even though we had integrated into our first simulations a GPS and also the description of the bike, the user was never able to find it as there were too many identical bicycles parked. We have then attached an acoustic signal tone to the smart-lock, that the user could activate. We have observed that this really improves the life of the user. We should not forget that this the smart-lock that relies on blockchain technology. However, if this technology is not "tangibilized", it remains useless, since nothing provided by the smart-lock is then perceived by the user. Typically, if the entire blueprint of the new concept is not systematically and rigorously simulated and thus not tested, it is not possible to get the new technology accepted [18].
ICNSER’20, 19-20 June, 2020, Kuala Lumpur, Malaysia

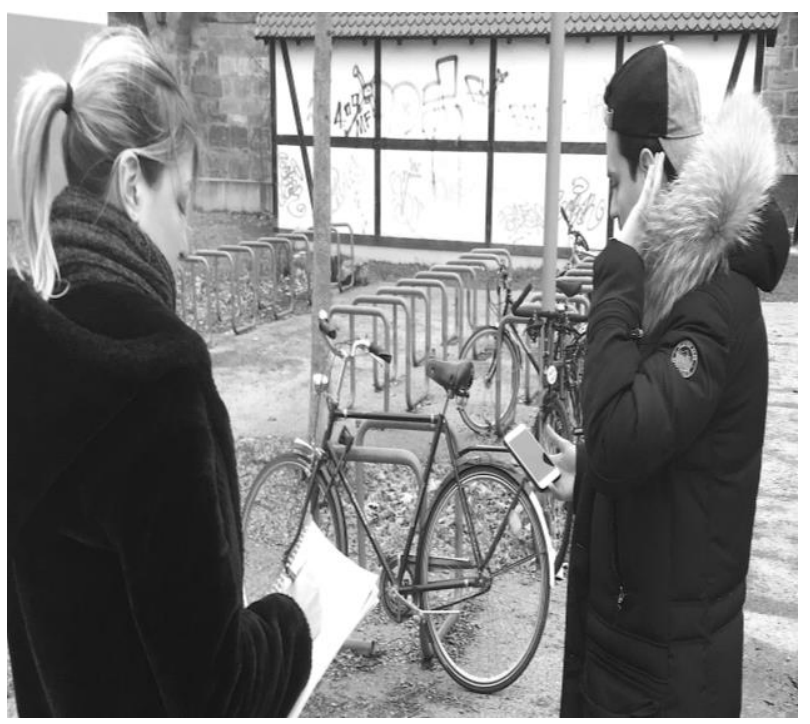

Figure 3: Photo extracted from the different videos used in postproduction to analyze our observations [11] (here acoustic test to find the borrowed bike)

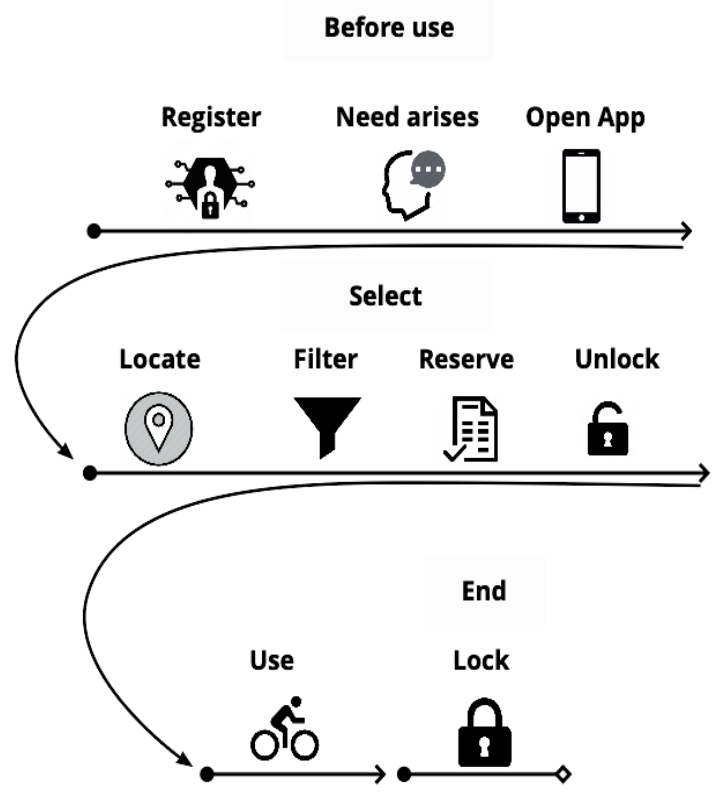

Figure 4: Customer Journey of the bike-sharing concept including the blockchain technology

\section{A Bike Sharing Model Based on Blockchain}

In this section, we develop an abstract model of bike-sharing service involving the blockchain technology with no middleman or service provider in-between. Our focus relies on the user, the adaption, and motivation to increase green transportation not just in dense cities, but also in the suburbs. 
Figure 5 shows a simplified model representation based on the concept of bike-sharing based on the blockchain technology. This development is based on both technological and logistical insights coming from our fieldwork and simulations presented in the previous section. Exchanges and interactions are happening directly between participants (i.e., C2C).

The exchange of bikes is based on the principle of smart contracts. A contract is no longer just concluded privately between two parties but is now publicly deposited on blockchains. This makes it easy to track who is supposed to have access and who is not - without a central provider. The blockchain becomes a kind of notary who officially approves every transaction. This interaction and exchange of assets are fully transparent but, at the same time, anonymous.

\section{USER to USER}

\section{BLOCKCHAIN}

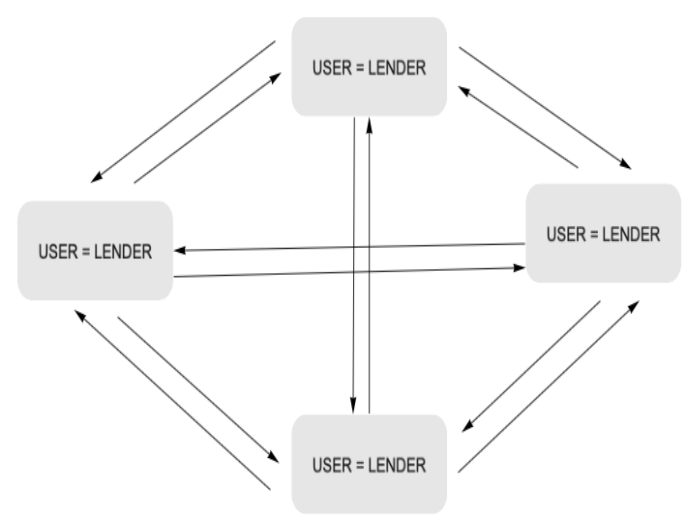

Figure 5: Bike-sharing model based on the blockchain technology

Another element to this concept development is then to consider what value we are going to propose to our customers. We have to define specifically what needs our bike-sharing model satisfies or/and what problem it solves. This concept relies then on a flexible, user-friendly peer-to-peer exchange of bikes directly between individuals. To eliminate dependency and to secure data privacy to a third party, we propose to implement this concept via smart contracts/blockchain as underlining technology. Therefore, our model allows a free and economical mode of transport between a restricted area for users who follow the current trends of a shared economy in order to adopt a more sustainable behavior in society.

\section{Conclusion}

In this paper, we have shown a methodology of service design to operationalize the blockchain technology in the actual process of renting out and using a bike without having any third party involved. Through the simulation staging of a series of hypothetical scenarios preventing a bike-sharing service to function properly, we have identified operational ways of improving the service experience for the user by relying as much as possible on the benefits provided by the blockchain technology. What is interesting with such "service design staging" is that no actual smart lock or app was used. Service design staging in such a prototyping phase can be used in an ex-ante manner, thus avoiding huge coding and implementation costs.

In order to move from a prototype, as presented in this article, to a dissemination of such an approach, it will be important to bring together all the professional actors to allow a real implementation of the blockchain technology through smart locks, self-governing identity and smart phones. The user is the engine of our concept. That is why we have focused essentially on service design approaches. As described in the article, "users are tired of using average or badly made products. Poor or loveless designed apps are no longer accepted so easily and often very quickly deleted from the mobile phone. In this case study, we have solely worked with one single persona. Therefore it will be highly recommended to enlarge the number of user-profiles and guide them through the processes we have done with Luis. Once we are satisfied with the results, we will target one specific city to perform a real-size experiment.

Indeed, the present research is exploratory and the corresponding contribution is therefore essentially methodological. We also intend, in subsequent research, to draw on previous quasi-experimental work [7] to validate the fail points identified in the process and the improvement solutions that we intend to design.

The blockhain is often seen as a purely IT issue. However, our simulations show that the effort required for the logistical and operational dimensions is enormous and, to our knowledge, insufficient today. Indeed, most of the effort is invested in coding and concepts. This is at least one thing we have learned from this applied research.

In connection with problems such as environmental pollution and mobility, it is now time to develop and implement new and innovative concepts based on the blockchain as part of the megatrend of the sharing economy.

In any case, the blockchain technology needs to be better explained to the public and should also require a profound redesign of any kind of service experience to have a real impact on society. Blockchain typically provides intangible benefits that have to be tangibilized through proper "experience staging".

\section{REFERENCES}

[1] A. Anjum, M. Sporny, M., and A. Sill (2017). Blockchain standards for compliance and trust. IEEE Cloud Computing, 4(4), 84-90.

[2]. R. Belk (2014). You are what you can access: Sharing and collaborative consumption online. Journal of business research, 67(8), 595-1600. 
[3] S. Benjaafar, S., G. Kong, X. Li, and C. Courcoubetis (2019) Peer-to-peer product sharing: Implications for ownership, usage, and social welfare in the sharing economy. Management Science, 65(2), 477-493.

[4] P. Bordalo, N. Gennaioli, A. Shleifer (2013).Salience and consumer choice. Journal of Political Economy, 121(5), 803-843.

[5] F. Casino, T. K. Dasaklis, and C. Patsakis (20219). A systematic literature review of blockchain-based applications: current status, classification and open issues. Telematics and Informatics, 36, 55-81.

[6] E.Fragnière, B. Nanchen, B., M. Sitten, M (2012). Performing service design experiments using ethnomethodology and theatre-based reenactment: a Swiss ski resort case study. Service Science, 4(2), 89-100.

[7] D. Gefen, and C. M. Ridings (2002). Implementation team responsiveness and user evaluation of customer relationship management: A quasi-experimental design study of social exchange theory. Journal of management information systems, 19(1), 47-69.

[8] F. Hawlitschek, B, Notheisen,, and T. Teubner (2018). The limits of trust-free systems: A literature review on blockchain technology and trust in the sharing economy. Electronic commerce research and applications, 29, 50-63.

[9] H. Heinrichs (2013). Sharing economy: a potential new pathway to sustainability. GAIA-Ecological Perspectives for Science and Society, 22, 228 231.

[10] M. Heuhert (2019). Conceptual Modeling Meets Customer Journey Mapping: Structuring a Tool for Service Innovation. In 2019 IEEE 21st Conference on Business Informatics (CBI), 1, 531-540.

[11] D. L. Jorgensen (1989). Participant observation: a methodology for human studies. Newbury Park (CA), Sage Publications.

[12] C. H. Lovelock (1996). Services marketing. Upper Saddle River, NJ: Prentice Hall.

[13] ,A. Mantelero (2015). Data protection, e-ticketing, and intelligent systems for public transport. International Data Privacy Law, 5(4), 309.

[14] P. Midgley (2009). The role of smart bike-sharing systems in urban mobility. Journeys, 2(1), 23-31.

[15] A. Pal, and Y. Zhang (2017). Free-floating bike sharing: Solving real-life largescale static rebalancing problems. Transportation Research Part C: Emerging Technologies, 80, 92-116.

[16] S. A. Shaheen, S. Guzman, and H. Zhang (2010). Bikesharing in Europe, the Americas, and Asia: past, present, and future. Transportation Research Record, 2143(1), 159-167.

[17] G. L. Shostack, (1993). How to design a service. European journal of Marketing, 16(1), 49-63.

[18] P. Surendran (2012). Technology acceptance model: A survey of literature. International Journal of Business and Social Research, 2(4), 175-178, 2012.

[19] S. L. Vargo, and R. F Lusch (2008). Service-dominant logic: continuing the evolution. Journal of the Academy of marketing Science, 36(1), 1-10.

[20] L.G. Zomerdijk, and C.A. Voss (2010). Service design for experience-centric services. Journal of service research, 13(1), 67-82. 